\title{
REGISTER RERUM ad Vol. 42
}

\section{Confecit G. Boehm, Basel}

Acetylcholinesterase, v. Skeletmuskel-Sehnenverbindung

Adaptations-Syndrom, v. Stress

Affe, v. Vertebral venous plexus

Age, v. Altersbestimmung

Akzessorische Sehne, v. Musculus abductor pollicis longus

Altersbestimmung der Katze, Bedeutung der Ossifikationspunkte (C) . . 262

Amniotic fluid, effect on the genital system of underfed rats

Anatomie von Aplodontia rufa rufa, v. Nagetiergehirn

Anatomie, mikroskopische, v. Histologie

Anatomie, topographische, v. Arteria maxillaris

Anatomie, vergleichende, v. Blutgefäße

Anatomistes Yougoslaves, comptes-rendus de la reunion annuelle (1959) (C) $261 \mathrm{ff}$.

Ane, v. Os

Aplodontia rufa rufa Rafinesque (= Biberhörnchen), v. Nagetiergehirn

Apparatus digestorius, v. Digestive tract

Arachnoid granulations in man, further studies

Ardour, phases, v. Hase

Arteria carotis communis, v. Carotid body

Arteria maxillaris; über die fraglichen Korrelationen und über die Entwick-

lung einiger Typen der Arteria maxillaris

71

-; Beitrag zu ihrer Kenntnis (C) 269

Arteria umbilicalis, v. Harnblase

Arterien der Harnblase, röntgenanatomische Untersuchung (C)

Ass, v. Os

Augenblase, v. Chick embryo

Autonomes Nervensystem, v. Sympathicus

Basophile, histiogene, der Maus (C) 269

Beef, v. Blutgefäße, Cervix uteri ruminantium

Bestrahlung, v. Irradiation, Radiokobalt, Röntgenstrahlen, X-rays

Biberhörnchen (= Aplodontia rufa rufa), v. Nagetiergehirn

Blood, v. Basophile, Haematopoetische Funktion

Blood, circulation, v. Mesenterialblutkreislauf, Sinus occipitalis

Blutgefäße und Nerven der vorderen Extremitäten bei Kamel, Lama, Gi

raffe, und Rind (C) 261

-; v. Gefäße

Blutkreislauf, v. Mesenterialblutkreislauf, Sinus occipitalis

Body, female, systematic morphology (C) 274

Bceuf, v. Blutgefäße, Cervix uteri ruminantium 
Bone,v. Clavicula, Os, Ossifikation, Ossifikationspunkte, Squama geographica

Book reviews (B) 175, 371

Bourse muqueuse, v. Musculus abductor pollicis longus Bovis, v. Blutgefäße, Cervix uteri ruminantuim Brain, v. Arachnoid granulations, Nagetiergehirn Brunst, Phasen, v. Hase

$1(\mathrm{~B})=$ Book reviews - Livres nouveaux - Buchbesprechungen $(C) \approx$ Report or demonstration Communication ou demonstration - Vor-trag oder Demonstration

Aeta anat.. Vol. 42, No. 4 (1960

24

374

Register rerum

Buchbesprechungen (B) $\quad 175,371$

Bursa, v. Musculus abductor pollicis longus

Cadavres, v. Nuclear sex determination

Calcium-Stoffwechsel; die Ossifikation im Lichte einer neuen Auffassung des

Calcium-Stoffwechsels (C) 265

Camel, v. Blutgefäße

Camelus bactrianus, v. Blutgefäße

Canal optique; les rapports du canal optique des enfants avec les sinus para-

nasaux postérieures (Contribution à $\Gamma$ étude du développement du sinus

sphénoïdal) (C) 272

-; v. in extenso: Acta anatomica, Vol. 41 (1960) 172

Canalis alimentarius, v. Digestive tract

Canís familíaris, v. Vascularisation, Vertebral venous plexus

Capillaires, v. Placenta

Capsule nasale, v. Nasenkapsel

Carotid body; the sinusoids of the carotid body tissue as part of the reti-

culo-endothelial system 213

Cartilage, hyaline; contribution to the study of the regenerative ability of

the hyaline cartilage (C) 264

Caryometry, v. Rein

Castor des montagnes (= Aplodontia rufa rufa), v. Nagetiergehirn

Cat, v. Katze

Cattle, horned cattle, v. Blutgefäße, Cervix uteri ruminantium

Cerebrum; Beiträge zur vergleichenden Morphologie des Nagetiergehirnes

(II.), Supplementum 40 (= 1 ad Vol. 42) Cerebrum, v. Arachnoid granulations Cerveau, v.

Arachnoid granulations, Cerebrum

Cervix uteri ruminantium, Feinbau und Schleimsekretion (C) 268

Chaleur (phases), v. Hase

Chameau, v. Blutgefäße

Chat, v. Katze

Cheval, v. Pancreas

Chick embryo; experimental production of retinal septa in the chick embryo.

Differentiation of pigment epithelium into neural retina 31

Chien, v. Vascularisation, Vertebral venous plexus

Children, v. Canal optique

Cholinesterase, v. Skeletmuskel-Sehnenverbindung 
Chondrocranium, v. Nasenkapsel

Chorionzotten; Beobachtungen über die Reifung der Chorionzotten in der menschlichen Placenta, mit besonderer Berücksichtigung der Epithel-

platten 12

Chromosomale Geschlechtsbestimmung, v. Sex chromatin Circulation intracranienne, v. Sinus occipitalis Circulation du sang, v. Mesenterialblutkreislauf

Clavicula; die Frühentwicklung der Clavicula beim Menschen $\quad 177$

Co60, v. Radiokobalt

Cobalt chloride, action on islets of Langerhans in horses (C) 272

Cobalt, radioactive, v. Radiokobalt

$\mathrm{CoC}^{1} \frac{1}{8}$, v. Cobalt chloride

Columna vertebralis, v. Venous plexus

Register rerum

375

Comparative anatomy, v. Blutgefäße

Comptes-rendus de la reunion annuelle des anatomistes Yougoslaves (1959) (C) $261 \mathrm{ff}$.

Congress, v. Comptes-rendus

Constitution, v. Female body

Corona dentis, v. Zahnkrone

Corps, structure, v. Female body

Corpse, v. Nuclear sex determination

Corpus pineale, v. Arachnoid granulations, Epiphysis cerebri

Corpuscule carotidien, v. Carotid body

Correlations, v. Arteria maxillaris

Corrigendum (ad Vol. 41 [1960] 187)

Couronne d'ivoire, v. Zahnkrone

Crane, v. Cranium

Cranium, v. Canal optique, Dural sinuses, Incisura tentorii, Nasenkapsel,

Sinus occipitalis, Squama geographica Cricetinae, v. Nagetiergehirn Crown of tooth, v.

Zahnkrone Cycle sexuel, v. Hase, Hypophysis

Cytochemistry, v. Histochemical effects, Skeletmuskel-Sehnenverbindung Cytotrophoblast, v.

Placenta

Darm, v. Nervous network

Daumen, v. Musculus abductor pollicis longus

Dead body, v. Nuclear sex determination

Deformations, v. Retinal septa, Tératogénèse

Deformities, v. Retinal septa, Tératogénèse

Degenerative changes, v. Amniotic fluid

Dentes, v. Dentin-...

Dentin-Kronenrelief; der Vergleich zwischen dem räumlichen Verhalten des

Dentin-Kronenreliefs und dem SchmelzreUef der Zahnkrone .... 226

Development, v. Canal optique, Clavicula

Diagnostik, mikroskopische, v. Histologie

Differentiation of pigment epithelium into neural retina. Experimental pro

duction of retinal septa in the chick embryo

Digestive tract; some characteristics in the fine structure of the main nervous 
network in the digestive tract (C) 264

Dog, v. Vascularisation, Vertebral venous plexus

Donkey, v. Os

Dura mater encephali, v. Incisura tentorii

Dural sinuses, confluence (C) 263

Eau douce, poissons, v. Haematopoetische Funktion

Eierstock der Häsin, Beitrag zur Kenntnis des Keimepithels (C) 262

Eierstock, v. Sex glands

Email dentaire, v. Schmelzrelief

Embryo, v. Clavicula, Nasenkapsel, Ontogenesis

Enamelum, v. Schmelzrelief

Enfants, v. Canal optique

Entwicklung, v. Arteria maxillaris, Frühentwicklung, Ontogenesis

Epididymis; l'infiuence d'excitations sonores sur l'épididyme de souris (C). . 276

Epiphysectomie, v. Epiphysis cerebri

376

Register rerum

Epiphysis cerebri; contribution à $\Gamma$ étude de $\Gamma$ histo-physiologie de la glande

thyroïde de rats épiphysectomisés $(\mathrm{C})$

-; influence de Гextrait épiphysaire sur la resistance à Гirradiation (C) . . 270

-; sur la réactivité stressogène du complexe habénulo-épiphysaire $(\mathrm{C})$. . 270

-; v. Arachnoid granulations

Epithalamus v. Habenula

Epithelium, v. Pigment epithelium

Epithelium germinative de Tovaire, v. Eierstock

Epithelplatten, v. Placenta

Equus asinus, v. Os

Equus caballus, v. Pancreas

Ernährung, v. Underfed rats

Erratum (ad Vol. 41 [1960] 187) 372

Esel, v. Os

Excitations sonores, influence sur Гépididyme de souris (C) 276

Experimental difformities, v. Retinal septa

Experimental goitre, v. Goitre

Extremitates anteriores, v. Blutgefäße, Musculus abductor pollicis longus

Eyes, v. Chick embryo

Facteurs tératogènes $(\mathrm{C})$

Fat, tissue, v. Female body Felís domestica, v. Katze

Female body, systematic morphology (C) 274

Ferments, v. Placenta, Skeletmuskel-Sehnenverbindung, X-rays

Fettgewebe, v. Female body

Fetus, v. Embryo

Fishs of fresh water, v. Haematopoetische Funktion

Forensic medicine; nuclear sex determination in forensic medicine .... 250

Fresh water; fishs of fresh water, v. Haematopoetische Funktion

Frühentwicklung der Clavicula beim Menschen 177 
Function and structure of muscle (Vol. I: Structure) (B) 175

Gallus gallus, v. Chick embryo

Gefäße des menschlichen Rückenmarks, Untersuchungen 277

Gefäße, v. Arachnoid granulations, Arteria maxillaris, Blutgefäße, Dural

sinuses, Glomus caroticum, Mesenterialblutkreislauf, Placenta, Sinus

occipitalis, Vascularisation, Vertebral venous plexus Gehirn; Beiträge zur vergleichenden

Morphologie des Nagetiergehirnes (II.),

Supplementum 40 (=1 ad Vol. 42) Gehirn, v. Arachnoid granulations

Genital system of underfed rats, effect of amniotic fluid 307

Gerichtliche Medizin, v. Nuclear sex determination

Germinal epithelium of the ovarium, v. Hase

Geschlechtsbestimmung, v. Sex chromatin

Geschlechtschromatin, v. Sex chromatin

Geschlechtsdrüsen, v. Sex glands

Gesellschaftsberichte, v. Anatomistes Yougoslaves

Gewebeanalyse, v. Female body

Giraffa catnelopardalis; die Glandula thyreoidea und die Glandulae thyreoi-

deae accessoriae bei Giraffa camelopardalis L. (C) 267

Register rerum 377

-; v. Blutgefäße

Giraffe, v. Gíraffa catnelopardalis

Glande pinéale, v. Arachnoid granulations, Epiphysis cerebri

Glande thyroïde, v. Thyreoidea

Glandes sexuelles, v. Sex glands

Glandula thyreoidea, und die Glandulae thyreoideae accessoriae bei Gíraffa

camelopardalis L. (C) 267

-; v. Goitre, Thyreoidea

Glatte Muskulatur; eine neue Auffassung über die Wirkung des Sympathicus

und des Parasympathicus auf die glatte Muskulatur, unter Berücksichti-

gung des anätomischen Substrates (C)

266

Glomus caroticum; the sinusoids of the carotid body tissue as part of the

re ticulo-endothelial system 213

Goitre, experimental; examinations of the structure of sex glands in animals

with experimental goitre (C) 271

Gravid ität, v. Placenta

Habenula; sur la réactivité stressogène du complexe habénulo-épiphysaire (C) 270

Haematopoetische Funktion der Nieren bei einigen Süßwasserfischarten (C) 266 Harnblase;

röntgenanatomische Untersuchung der Arterien der Harnblase (C) 268 Harnblasenschleimhaut,

Regeneration, Wirkung subletaler Dosen von X-

Strahlen $(C) \quad 271$

Hase; Beitrag zur Kenntnis des Keimepithels am Eierstock der Häsin (C) 262 Hemionus Kiang

Kíang Moocroft, v. Os Hiatus tentorii, v. Incisura tentorii

Histiogene Basophile der Maus (C) 269

Histochemical effects of $\mathrm{X}$ radiation 1

Histochemistry, v. Skeletmuskel-Sehnenverbindung

Histologie und mikroskopische Anatomie des Menschen mit Berücksichti- 
gung der Histophysiologie und der mikroskopischen Diagnostik (2.

Aufl.)(B) $\quad 371$

Histophysiologie, v. Histologie

Hoden; histomorphologische Veränderungen an Mäusehoden nach Radio-

kobalt-Bestrahlung (C) 270

$-; \mathrm{v}$. Sex glands

Homme, v. Man

Horse, v. Pancreas

Hühnchen, v. Chick embryo

Humain, clavicule humain, v. Clavicula

Human embryo, v. Nasenkapsel

Hund, v. Vascularisation, Vertebral venous plexus

Hyaline cartilage; contribution to the study of the regenerative ability of the

hyaline cartilage (C) 264

Hypophysektomie, Auswirkung auf das lymphatische Gewebe von Ratten (C) 275 Hypophysis;

der Sekretionszustand der Hypophyse des Zanders (Lucioperca

sandra) im Verlaufe des Sexualzyklus (C) 274

Incisura tentorii; über die Morphologie und die Beziehungen der Incisura

tentorii (C) . . 263

Intestinum, v. Nervous network

Intracranienne, circulation intracranienne, v. Sinus occipitaüs

378

Register rerum

Irradition; influence de $\Gamma$ extrait épiphysaire sur la resistance à l'irradiation

(C) 270

-; v. Radiation, Radiokobalt, Röntgenstrahlen, Thyreoidea Isotope, radioaktive, v. Radiokobalt

Jonction musculo-tendineuse, v. Skeletmuskel-Sehnenverbindung

Kalzium-Stoffwechsel, v. Calcium-Stoffwechsel Kamel, v. Blutgefäße Kapillaren, v. Placenta

Karyometrie, v. Rein

Katze, Altersbestimmung, Bedeutung der Ossifikationspunkte (C) 262

Keimepithel am Eierstock der Häsin, Beitrag zu seiner Kenntnis (C) . . 262

Kerne, v. Rein

Kiang, v. Os

Kidney, v. Niere, Rein

Kinder, v. Canal optique

Knochen, v. Clavicula, Os, Ossifikation, Ossifikationspunkte, Squama geo-

graphica Knorpel, v. Cartilage Kobalt, radioaktives, v. Radiokobalt Kobaltchlorid, v. Cobalt

chloride Körperbau, v. Female body Kongress, v. Anatomistes Yougoslaves Konstitution, v.

Female body Korrelationen, v. Arteria maxillaris Kropf, experimenteller, v. Goitre

Lama, v. Blutgefäße

Lama glama, v. Blutgefäße

Langerhans, islets of Langerhans, v. Pancreas

Leichen, v. Nuclear sex determination

Lepus europaeus, v. Hase

Livres nouveaux (B) 175,371

Llama, v. Blutgefäße 
Lucioperca sandra, v. Hypophysis

Lymphatisches Gewebe von Ratten, Auswirkung der Hypophysektomie (C) 275

Macula densa du rein 352

Malformations, v. Tératogénèse Malformations oculaires, v. Chick embryo

Man; arachnoid granulations in man, further studies $\quad 88$

-; die Frühentwicklung der Clavicula beim Menschen 177

-; die frühe Morphogenesis der Nasenkapsel beim Menschen

Man, v. Arteria maxillaris, Histologie, Placenta, Rückenmark

Mandibula, v. Prominentiae osseae transitoriae

Mast cells, v. Histiogene Basophile

Mastozyten, v. Histiogene Basophile

Maturation placentaire, v. Reifung der Chorionzotten

Maus; histomorphologische Veränderungen an Mäusehoden nach Radio-

kobalt-Bestrahlung (C) 270

-; v. Epididymis, Histiogene Basophile

Register rerum

379

Maxilla, v. Prominentiae osseae

Médecine judiciaire, v. Nuclear sex determination

Medulla spinalis, v. Rückenmark, Venous plexus

Membra superiores, v. Blutgefäße, Musculus abductor pollicis longus

Meninges, v. Arachnoid granulations, Incisura tentorii

Mensch; die Frühentwicklung der Clavicula beim Menschen $\quad 177$

-; die friihe Morphogenesis der Nasenkapsel beim Menschen 105

-; v. Histologie, Man, Rückenmark

Mesenterialblutkreislauf der weißen Ratte, Einfluß der Temperatur (Film)

(C) 269

Metabolismus, v. Calcium-Stoffwechsel

Mice, v. Epididymis, Histiogene Basophile, Testes

Mícrotinae, v. Nagetiergehirn

Mikroskopische Anatomie, v. Histologie

Mikroskopische Diagnostik, v. Histologie

Mißbildungen, v. Retinal septa, Tératogénèse

Moelle épinière, v. Rückenmark, Venous plexus

Monkey, v. Vertebral venous plexus

Morphogenesis, frühe, der Nasenkapsel beim Menschen 105

-; v. Clavicula, Ontogenesis, Retinal septa

Morphology; systematic morphology of the female body (C)

Mountain beaver (= Aplodontia rufa rufa), v. Nagetiergehirn

Mouse, v. Epididymis, Histiogene Basophile, Testes

Mucosa, v. Harnblasenschleimhaut

Mucosa-Epithelzellen, v. Cervix uteri ruminantium

Mucous membrane of the urinary bladder, v. Harnblasenschleimhaut

Mucus, secretion of mucus, v. Cervix uteri ruminantium

Muqueuse de la vessie urinaire, v. Harnblasenschleimhaut

Murínae, v. Nagetiergehirn 
Mus musculus, v. Epididymis, Histiogene Basophile, Testes

Muscle; structure and function of muscle (Vol. I: Structure) (B)

Muscle, v. Muskel-Sehnenverbindung

Muscle, v. Skeletmuskel-Sehnenverbindung

Muscles, smooth, v. Glatte Muskulatur

Musculature lisse, v. Glatte Muskulatur

Musculus abductor pollicis longus (Seine akzessorische Sehne und Bursa) (C) 265

Musculus pterygoideus, v. Arteria maxillaris

Muskel-Sehnenverbindung; Untersuchungen über die Skeletmuskel-Sehnen

verbindung (II.: Histochemische Lokalisation der Acetylcholinesterase

und Untersuchungen über ihre mögliche Funktion an der Muskelfaser-

Sehnenverbindung 318

Muskel, v. Muscle

Muskulatur, glatte, v. Glatte Muskulatur

Myomorpha, v. Nagetiergehirn

Myo-tendinous junctions, v. Skeletmuskel-Sehnenverbindung

Nagetiergehirn; Beiträge zur vergleichenden Morphologie des Nagetierge-

hirnes (II.), Supplementum 40 (= 1 ad Vol. 42) Nasal capsule, v. Nasenkapsel Nasenkapsel;

frühe Morphogenesis der Nasenkapsel beim Menschen. . . . 105

380

Register rerum

Nasus, v. Nasenkapsel

Nephron, v. Macula densa

Nerven und Blutgefäße der vorderen Extremitäten bei Kamel, Lama, Giraffe

und Rind (C) 261

Nerven (Nervensystem, Zentralnervensystem), v. Arachnoid granulations,

Nagetiergehirn, Nervous network, Nervus, Retinal septa, Sympathicus Nervous network; some characteristics in the fine structure of the main

nervous network in the digestive tract (C) 264

Nervus mandibularis, v. Arteria maxillaris

Neural retina, v. Retinal septa

Nez, v. Nasenkapsel

Niere; die haematopoetische Funktion der Niere bei einigen Süßwasserfisch-

$\operatorname{arten}(\mathrm{C}) \quad 266$

- ; v. Rein

Nose, v. Nasenkapsel

Nourriture insuffisante, $v$. Underfed rats

Noyaux, v. Rein

Nuclear sex determination in forensic medicine $\quad 250$

Nuclei, v. Rein

Nutrition, v. Underfed rats

CEil, v. Chick embryo

Oestrus, cycle, v. Hase, Hypophysis

Ontogenesis; die frühe Morphogenesis der Nasenkapsel beim Menschen . . 105

-; die Frühentwicklung der Clavicula beim Menschen 177

-; v. Arteria maxillaris, Placenta, Retinal septa 
Optic vesicle, v. Chick embryo

Organa genitalia feminina, v. Underfed rats

Os; prominentiae osseae transitoriae mandibulares et maxillares bei Equus

asinus und Hemionus Kiang Kíang Moorcroft (C) 273

-; v. Canal optique, Clavicula, Ossifikation, Ossifikationspunkte

Os temporale, v. Squama geographica

Ossifikation, im Lichte einer neuen Auffassung des Calcium-Stoffwechsels (C) 265

-; v. Clavicula

Ossifikationspunkte, Bedeutung für die Altersbestimmung der Katze (C) . . 262

Ovarium; Beitrag zur Kenntnis des Keimepithels am Eierstock der Häsin (C) 262

-; v. Genital system, Sex glands

Pancreas; action of cobalt chloride on islets of Langerhans in horse (C) . . 272

Parasympathicus und Sympathicus; neue Auffassung über ihre Wirkung auf

die glatte Muskulatur, unter Berücksichtigung des anatomischen

Substrates (C) 266

Pferd, v. Pancreas

Physiologie (Histophysiologie), v. Histologie

Pigment epithelium into neural retina, differentiation. Experimental pro

duction of retinal septa in the chick embryo 31

Pike-perch, v. Hypophysis

Pineal body, v. Arachnoid granulations

Pisces, v. Haematopoetische Funktion

Register rerum 381

Pituitary, v. Hypophysektomie, Hypophysis

Placenta; Beobachtungen über die Reifung der Chorionzotten in der mensch-

lichen Placenta, mit besonderer Berücksichtigung der Epithelplatten 12 Plaque dense, v. Rein

Plexus myentericus; some characteristics in the fine structure of the main

nervous network in the digestive tract (C) 264

Poissons de l'eau douce, v. Haematopoetische Funktion

Pouce, v. Musculus abductor pollicis longus

Poulet, v. Chick embryo

Prominentiae osseae transitoriae mandibulares et maxillares bei Equus asínus

und Hemionus Kiang Kiang Moorcroft (C) 273

Radiation; histochemical effects of X-radiation .

-; v. Irradiation, Radiokobalt, Röntgenstrahlen, Thyreoidea

Radiokobalt; histomorphologische Veränderungen an Mäusehoden nach

Radiokobalt-Bestrahlung (C) 270

Rat; effect of amniotic fluid on the genital system of underfed rats .... 307

-; v. Epiphysis cerebri, Goitre, Hypophysektomie, Mesenterialbl·utkreislauf, Thyreoidea

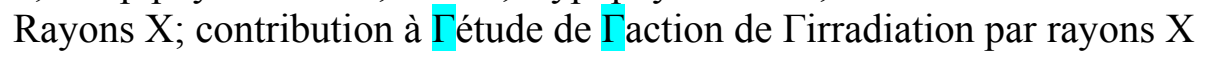

sur la glande thyroïde (C) 275

-; v. Epiphysis cerebri, Röntgenanatomische Untersuchung, Röntgenstrahlen, X-rays

Regeneration; contribution to the study of the regenerative ability of the

hyaline cartilage (C) 264

Regeneration der Harnblasenschleimhaut, Wirkung subletaler Dosen von

X-Strahlen (C) 271 
Reifung der Chorionzotten in der menschlichen Placenta, mit besonderer Be rücksichtigung der Epithelplatten, Beobachtungen

Rein; contribution à I'étude de la vascularisation du rein chez le chien (C) 261

-; à propos de la macula densa du rein .352

$-;$ v. Niere

Ren, v. Niere, Rein, Vascularisation

Reticulo-endothelial system; the sinusoids of the carotid body tissue as part

of the reticulo-endothelial system 213

Retinal septa; experimental production of retinal septa in the chick embryo.

Differentiation of pigment epithelium into neural retina 31

Rind, v. Blutgefäße, Cervix uteri ruminantium

Rodentía, v. Nagetiergehirn

Röntgenanatomische Untersuchung der Arterien der Harnblase (C) .... 268

Röntgenstrahlen; Wirkung subletaler Dosen von X-Strahlen auf die Regene ration der Harnblasenschleimhaut (C)

-; v. Epiphysis cerebri, Rayons X, X-rays

Rongeurs, v. Nagetiergehirn

Rückenmark; Untersuchungen über die Gefäße des menschlichen Rücken-

marks 277

-; v. Venous plexus

Ruminants, v. Cervix uteri ruminantium

Ruminatores, v. Cervix uteri ruminantium

382

Register rerum

Sandre, v. Hypophysis

Schädel, v. Canal optique, Dural sinuses, Incisura tentorii, Nasenkapsel, Sinus occipitalis,

Squama geographica

Schall, Einwirkung, v. Epididymis

Schilddrüse, v. Thyreoidea

Schleimbeutel, v. Musculus abductor pollicis longus

Schleimhaut; Wirkung subletaler Dosen von X-Strahlen auf die Regeneration der Harnblasenschleimhaut (C) 271

Schleimsekretion, v. Cervix uteri ruminantium

Schmelzrelief der Zahnkrone; der Vergleich zwischen dem räumlichen Verhalten des Dentin-Kronenreliefs und dem Schmelzrelief der Zahnkrone 226

Scíuromorpha, v. Nagetiergehirn

Secretion muqueuse, v. Cervix uteri ruminantium

Sehne, akzessorische, v. Musculus abductor pollicis longus

Sehne; Skeletmuskel-Sehnenverbindung (II.: Histochemische Lokalisation

der Acetylcholinesterase und Untersuchungen über ihre mögliche

Funktion an der Muskelfaser-Sehnenverbindung) 318

Sewelled beaver (= Aplodontía rufa rufá), v. Nagetiergehirn

Sex chromatin; nuclear sex determination in forensic medicine

Sexualzyklus, v. Hase, Hypophysis

Sex glands; examination of the structure of sex glands in animals with ex perimental goitre (C) 271 
-; v. Genital system

Singe, v. Vertebral venous plexus

Sinus caroticus; the sinusoids of the carotid body tissue as part of the reti

culo-endothelial system 213

Sinus cranien, v. Sinus durae matris

Sinus durae matris; the confluence of dural sinuses (C) 263

Sinus occipitalis; Гimportance du sinus occipital pour le rétablissement de

la circulation intracranienne de retour après la resection bilatérale des

deux jugulaires internes (C) 274

Sinus paranasaux; les rapports du canal optique des enfants avec les sinus

paranasaux postérieures (Contributions au développement du sinus

sphénoïdal) (C) 272

-; v. in extenso: Acta anatomica Vol. 41 (1961) 172

Sinus sphenoidalis, v. Sinus paranasaux

Sinusoids of the carotid body tissue as part of the reticulo-endothelial system 213

Sitzungsberichte, v. Anatomistes Yougoslaves

Skeletmuskel-Sehnenverbindung, Untersuchungen (II.: Histochemische

Lokalisation der Acetylcholinesterase und Untersuchungen über ihre

mögliche Funktion an der Muskelfaser-Sehnenverbindung) 318

Skull, v. Canal optique, Dural sinuses, Incisura tentorii, Nasenkapsel, Sinus occipitalis, Squama geographica

Société, v. Anatomistes Yougoslaves

Society transactions, v. Anatomistes Yougoslaves

Son, influence, v. Epididymis

Sound, effects, v. Epididymis

Souris, v. Epididymis, Histiogene Basophile, Testes

Spinal cord, v. Rückenmark, Venous plexus

Splanchnologie von Aplodontia rufa rufa, v. Nagetiergehirn

Register rerum

383

Squama geographica (C) 273

Squama temporalis, v. Squama geographica

Statistische Auswertung, v. Arteria maxillaris

Stoffwechsel, v. Calcium-Stoffwechsel

Stress; sur la réactivité stressogène du complexe habénulo-épiphysaire (C) 270

Structure and function of muscle (Vol. I: Structure) (B) 175

Subcutaneous fat, v. Female body

Substantia adamantina, v. Schmelzrelief

Süßwasserfischarten, v. Haematopoetische Funktion

Sympathicus und Parasympathicus; neue Auffassung über ihre Wirkung auf

die glatte Muskulatur, unter Berücksichtigung des anatomischen Sub

strates (C) 266

Syncytiotrophoblast, v. Placenta

Syncytium, v. Placenta

Synovial bursa, v. Musculus abductor pollicis longus

Systema digestorium, v. Digestive tract 
Taxonomie, v. Nagetiergehirn

Teeth, v. Dentin-...

Temperatur, Einfluß auf den Mesenterialblutkreislauf der weißen Ratte

(Film)(C) 269

Tendon, v. Musculus abductor pollicis longus

Tendon (myo-tendinous junctions), v. Skeletmuskel-Sehnenverbindung

Teratogénèse; facteurs tératogènes $(\mathrm{C}) \quad 267$

Teratologie, v. Retinal septa

Testes; histomorphologische Veränderungen an Mäusehoden nach Radio-

kobalt-Bestrahlung (C) 270

- ; v. Sex glands

Thumb, v. Musculus abductor pollicis longus

Thyreoidea; contribution à $\Gamma$ étude de Faction de Tirradiation par rayons $\mathrm{X}$

sur la glande thyroïde (C) 275

-; contribution à l'étude de l'histo-physiologie de la glande thyroïde de rats

épiphysectomisés (C) 273

-; v. Glandula thyreoidea, Goitre

Tissu adipeux, v. Female body

Tone, Einwirkung, v. Epididymis

Tooth, v. Dentin-...

Topographische Anatomie, v. Arteria maxillaris

Transactions, v. Anatomistes Yougoslaves

Tube digestif, v. Digestive tract

Tubes urinifères, $v$. Rein

Tubuli renales contorti, v. Rein

Tunica mucosa vesicae urinariae, v. Harnblasenschleimhaut

Underfed rats; effect of amniotic fluid on the genital system of underfed rats 307

Unterernährte Ratten, v. Underfed rats

Urinary bladder, v. Arterien, Harnblasenschleimhaut

Uterus; über den Feinbau und die Schleimsekretion der Cervix uteri rumi-

nantium (C) 267

384

Register rerum

Vaisseaux, v. Arachnoid granulations, Arteria maxillaris, Blutgefäße, Dural sinuses, Glomus caroticum, Mesenterialblutkreislauf, Placenta, Rücken-mark, Sinus occipitalis, Vascularisation, Vertebral venous plexus

Variationen, v. Arteria maxillaris

Vascularisation du rein chez le chien, contribution à son etude (C) .... 261

Vegetatives Nervensystem, v. Sympathicus

Veins, v. Arachnoid granulations, Dural sinuses, Sinus occipitalis, Venous plexus

Venae jugulares internae (resection bilatérale), v. Sinus occipitalis

Venen, v. Arachnoid granulations, Dural sinuses, Sinus occipitalis, Venous plexus

Venous plexus, vertebral 333

Verdauungskanal, v. Digestive tract

Vergleichende Anatomie, v. Blutgefäße, Nagetiergehirn

Vergleichende Anatomie, v. Blutgefäße 
Versammlungsberichte, v. Anatomistes Yougoslaves

Vertebral venous plexus 333

Vesica urinaria, v. Arterien, Harnblasenschleimhaut

Vésicule optique, v. Chick embryo

Vessels, v. Arachnoid granulations, Arteria maxillaris, Blutgefäße, Dural sinuses, Glomus

caroticum, Mesenterialblutkreislauf, Placenta, Rücken-mark, Sinus occipitalis, Vascularisation, Vertebral venous plexus

Vessie (urinaire), v. Arterien, Harnblasenschleimhaut

Viscères de Aplodontia rufa rufa, v. Nagetiergehirn

Vordere Extremitäten, v. Blutgefäße

Weiblicher Körper, v. Female body Wïederkäuer, v. Cervix uteri ruminantium Wirbelsäule, v.

Vertebral venous plexus

$\mathrm{X}$-rays; histochemical effects of X-radiation

X-rays, v. Epiphysis cerebri, Röntgenanatomische Untersuchung, Rönt-genstrahlen, Thyreoidea Yougoslavie; comptes-rendus de la reunion des anatomistes Yougoslaves

(1959) (C) $261 \mathrm{ff}$.

Zahnkrone; der Vergleich zwischen dem räumlichen Verhalten des Dentin-

Kronenreliefs und dem Schmelzrelief der Zahnkrone

226

Zander, v. Hypophysis Zirbeldrüse, v. Epiphysis cerebri

Book Reviews - Livres Nouveaux - Buchbesprechungen

Bourne, G. H. (Editor): The structure and function of muscle (Vol. I: Struc

ture) (Academic Press Inc., New York 1960) 175

Bucher, 0.: Histologie und mikroskopische Anatomie des Menschen mit Be-rücksichtigung der Histophysiologie und der mikroskopischen Dia-gnostik (2. Aufl.) (Medizinischer Verlag Hans Huber, Bern 1956) . . 371 